\title{
The Applications of Digital Multimedia in Mobile Game
}

\author{
Tang Qianjun, Zhang Yan \\ Leshan Normal University, Leshan, Sichuan, 614000 \\ 57225351@qq.com
}

Keyword: mobile games; digital multimedia; technical thinking

\begin{abstract}
With the continuous acceleration of social development, information technology has been constantly innovated and developed. Digital multimedia has become a new form of artistic expression under the background of information technology. It effectively integrates rational and artistic thinking, and exists in all walks of life in a comprehensive way of art. By launching a series of explorations of digital multimedia technology in mobile games, this research gives a brief overview of digital multimedia theory and main artistic features, analyzes the main development process and characteristics of mobile games, and finally explores how to apply the digital multimedia technology to the mobile game more efficiently, to improve mobile games for better production results. This study is expected to provide a theoretical basis for the research and development of mobile games in China.
\end{abstract}

\section{Introduction}

With the rapid development of the information age accelerating, the development of digital information technology, network technology and cultural industries is also accelerating. The digital multimedia art industry has become a new form of industry in the current society. Digital multimedia is widely integrated in all walks of life, and now it has become the core industry of the main knowledge economy under the development of the current times. With the rapid development of digital multimedia in recent years, many new technologies and products have been deeply developed and widely used. Digital multimedia art mainly comes from the computer terminal equipment. It's a innovation process of the producers who finally finish the art works through expressing their own artistic thinking in many ways. And it is a process of artistic and aesthetic popularization that is widely popularized by the use of digital related media. From the perspective of disciplinary development, digital multimedia art is a new form of art based on digital media, and a comprehensive interdisciplinary subject of art and technology. Therefore, this study launched a series of research aimed at the relationship between digital multimedia technology and mobile games.

\section{Features of digital multimedia art}

Features of creative tools. The form of digital multimedia art is based on computer equipment and Internet technology. And it has the obvious characteristics of artistic creation tools. In other words, digital multimedia art is a kind of integrated digital technology existing in the subject knowledge of painters and musicians. While, this technical form is mainly highlighted by digital technology and needs to be highlighted by digital technology equipment. The characteristics of the creation tool can effectively break the traditional way of creation. It can be better realized with the help of innovative research and development of new technology. However, the characteristics of the creative tools of digital multimedia art are different from the traditional art forms. The artistic creation mode of digital multimedia and the corresponding influence of the process of formation all show the characteristics of the creation tool of digital multimedia art to a great extent. In a word, multimedia art has largely broken through the prominent tool of traditional art, especially the powerful internet technology support. 
Media Features. The artistic features of digital multimedia are also characterized by media characteristics. The specific features are manifested in the overall communication process of the digital multimedia art works. The main ways of dissemination of multimedia art works in current digital multimedia platforms including radio, magazines, musical record, newspapers and television, the independent digital media mainly include CD and USB media etc.. It is particularly important that designers can use the Internet platform to instantly complete the sharing of their own works. Such as video sites and music sites, and the overall audience can effectively communicate with the relevant designers. To get the appropriate immediate feedback, ,the two-way communication process of thinking is effectively realized.

Virtual features. The artistic features of digital multimedia also have virtual features. This feature is a virtual expression mainly that is based on the diverse ornamental of the designer and the appreciator to effectively combine the thinking and the reality . The traditional art is still unable to match the specific advantages of digital multimedia art in the creation. The digital multimedia art creation is based on the digital media equipment, which can effectively achieve something that the traditional way of creation can not achieve. For example, if a certain error occurs during the painting process, the history function in the digital software technology can be effectively used, the corresponding operation can be effectively returned and modified, and the effectiveness of the scene production operation can be observed more effectively. In addition, digital multimedia art also has a certain degree of interactivity, which can effectively complete the corresponding interactive function browsing, reflected by the relevant media features.

\section{The characteristics of the development process of mobile game}

In the early stages of mobile games, the game was mainly written in the form of text. This kind of game is a game process that the players complete the corresponding information response by using mobile phones. For example, the earlier "virtual pet" mobile games belongs to the more typical mobile word game. During such a game, the game's operation service merchants can give appropriate prompts and then complete the text-based game process via SMS. The existence of this game can effectively use text messages to complete the communications of games to achieve the purpose of the game entertainment. As the whole process of the game is expressed through the text, the overall entertainment of the game is relatively poor. Therefore, during the game, players need to think in accordance with their own game thinking to enhance the fun of the game.

With the continuous innovation and development of information technology in recent years, the functionality of mobile phones is becoming more and more abundant. Through the application of digital multimedia in mobile phone games, we can enhance the sense of gaming experience of mobile phones, making mobile games more entertaining and interactive. Currently, mobile games are usually animated games, such as "PC games", and the games are completed through animated plot types. Mobile games are usually more intuitive and beautiful to enhance the visual entertainment of the screen. Therefore, animation mobile games are more popular with players. However, different types of handsets have larger functional differences in overall device functionality. Therefore, in the development of the game, it is necessary to balance the related factors more efficiently. For example, considering the resolution of mobile phones. Because the screens size of the different mobile phone is different, the resolution of the screen is also different. At the same time, the system function of different types of mobile phones has brought some changes to the operation of the game. The impact of the screen color on the mobile phone is relatively less than the overall display of the computer device, and it is also an impact on the design workers of the game. And the impact of the screen brightness of the mobile phone will lead to colorful patterns, to show better application results in a more intense colors. For example, mobile phone users will be more obvious in the outdoors. Design workers must also consider this point in the design of the game, so that the overall design of the game is more concise. Through the consideration of many aspects of the characteristics of the impact to better integrate digital multimedia and mobile games. 


\section{Digital multimedia advances the progress of mobile games}

Nowadays, the entertainment industry has become a new type of economic growth point, and this situation is not an exception in the current development process of the entertainment industry in China. The development of the entertainment industry in China is particularly prominent, which is based on digital multimedia technology, and the new form of artistic expression is being gradually constructed. Mobile games have also spread all over the current mobile phone users, and are also places where digital multimedia is used for entertainment. For example, after we have purchased a new collection, we will find more and more wonderful games. Mobile games have become an important part of most people's lives and people are also more accustomed to using mobile games as a new type of entertainment. In the process of making mobile games, better integration of computer technology and digital multimedia technology is achieved. The most often used is the design tools and the common computer game software. The vast majority of mobile game production processes that are in use typically make secondary edits after modeling with 3dsMx and Maya. Construction is also especially important in the overall production of mobile games. Part of the overall image can not be effectively described in detail.

For some designers, although the overall picture of the mobile phone game and the character movement design relatively need to be as delicate and complicated as possible it is usually difficult on mobile phone games. Because of the more delicate and complex animation will inevitably occupy a mass collect storage space. No player wants to be able to play the game in spite of the other functions of the mobile phone. So in the process of making mobile games, it is usually necessary to reduce the number of frames as much as possible to continuously enhance the overall reuse of the frame. As much as possible to highlight the overall animation action to achieve better mobile animation game results.

In a word, the application of digital multimedia in mobile game creation is very important and essential. In the process of making mobile games today, the use of digital multimedia technology is usually more and more inescapable. In today's mobile games, the artistic features of digital multimedia can be seen everywhere. For example, the passing of missiles, the pursuit of cars, the fighting between characters, and the war of soldiers are all showing the strength of digital multimedia art everywhere. Based on the purely technical point of view, the digital multimedia art has greatly changed the whole design process of mobile games. With the help of computers, we can achieve better creation of mobile phone games. It can effectively combine images and picture sounds, and has large storage capacity, the overall display process is faster, and it can be modified and released at any time. Based on the overall design progress, it can save much time for designers, so that designers can better concentrate more energy and focus their overall thinking on strategy and creative conception.

The development of digital multimedia technology has become an important technical support for the research and development of mobile games. Its existence means that the creation of mobile games is more diversified and modernized, and it meets more and more users' needs for diverse pages, functions and visual experience of games. As mobile games and digital multimedia technologies continue to be highly innovated and integrated, there are more innovative methods and overall production methods. Digital multimedia art is the most efficient, all round and comprehensive multiple platform production application in the overall development of mobile games. At the same time, it can effectively make mobile games adapt to the continuous innovation of the modern social technology and development, embodying in a more diverse form. Digital multimedia and mobile phone games are more objective in further social development process, and are better achieved through continuous technological innovation and artistic thinking collision.

\section{Acknowledgements}

The thesis was funded by the following grant projects: Science and Technology Bureau of Lesha n City-Construct multimedia educational database in the computing cloudy situation(14JK013); Th -e 2nd pilot comprehensive education reform project in Sichuan province- depth integration of mod 
ern education technology into the primary and lower secondary school education.

\section{Reference}

[1] Liu Yunzhi. Innovative Application of Digital Media Technology in Games[J].Electronic Technology and Software Engineering, 2017(11):83-83.

[2] Sun Yuning. On the Application of Technology in the Development of Digital Media Art Games[J]. Modern decoration: theory, 2015(11).

[3] Wu Guangzhi. Technical Application Analysis of Digital Media Art Game Production[J]. Technology Communication, 2015(3).

[4] Zhang Jianchun. Application of "Third Perspective" in Digital Media Games[J]. Art and Design: Theoretical Edition, 2014(5):88-89.

[5] Tang Ni. Research on APP Application of Digital Museum Based on Mobile Media - A Case Study of "Sponge City" in Chengdu Museum[D]. Southwest Jiao Tong University, 2017.

[6] Punt M, Bjelica M Z, Zdravkovic V, et al. An integrated environment and development framework for social gaming using mobile devices, digital TV and Internet[J]. Multimedia Tools \& Applications, 2015, 74(18):8137-8169.

[7] Feng Zhigang, Wang Chunyan. The Application of Contemporary New Media Digital Technology[J]. Chinese Media Science and Technology, 2013(20).

[8] Sun Lu. Brief Analysis on the Utilization and Influence of Digital Art for Video Special Effects and Digital Games[J]. JinTian, 2013(2). 